\title{
El académico cuantificado. La gestión performativa a través de los instrumentos de medición en la ciencia
}

\author{
The quantified academic. Performative \\ management through measurement instruments \\ in science
}

\section{O acadêmico quantificado. Gestão performativa através de instrumentos de medição em ciência}

Carla Fardella Cisternas

ORCID ID: 0000-0001-8936-2435

Facultad de Educación y Ciencias Sociales

Universidad Andrés Bello, Chile

Alejandra Paz Corvalán Navia ORCID ID: 0000-0002-6329-403X

Facultad de Educación y Ciencias Sociales Universidad Andrés Bello, Chile

Ricardo Zavala Villegas ORCID ID: 0000-0001-9042-7822 Facultad de Educación y Ciencias Sociales Universidad Andrés Bello, Chile

Autor referente: carla.fardella@unab.cl

Historia editorial Recibido: 06/04/2019 Aceptado: 03/09/2019

\section{RESUMEN}

Este artículo reflexiona sobre la subjetividad de los académicos a partir de un análisis cualitativo empírico de

los instrumentos de acción pública para gestionar la producción científica en Chile. El presente estudio propone que 
los dispositivos de gestión, no sólo buscan optimizar la producción científica nacional, sino también tiene importantes efectos sobre quienes desarrollan el conocimiento. Con el objetivo de indagar en esto se realizó un análisis discursivo de 200 documentos públicos y oficiales, los cuales reúnen bases de concurso para adjudicación de becas, formularios e instructivos para distribuir recursos para investigar. Los documentos analizados son comprendidos como una representación de la gestión pública de la ciencia, pero sobre todo como objetos ordenan y performan el mundo científico. Tras un análisis discursivo de los documentos podemos mostrar cómo los instrumentos trazan el desarrollo de un "yo académico cuantificado"; que requiere que los académicos se involucren activamente, mediante el registro de sus actividades bajo el lenguaje de la medición y cuantificación. Sin duda, la traducción de la actividad científica a este tipo dispositivos juega un rol trascendental, transformando las vidas científicas. El artículo muestra cómo los documentos actúan sobre la subjetividad y concluye reiterando la necesidad de un uso y compromiso crítico con las tecnologías de gestión en las políticas públicas científicas.

Palabras clave: Instrumentos de gestión; subjetividad laboral; capitalismo académico

\section{ABSTRACT}

This article reflects on the subjectivity of academics based on a qualitative empirical analysis of the instruments of public action to manage scientific production in Chile. This study proposes that management devices not only seek to optimize national scientific production, but also have important effects on those who develop knowledge. In order to investigate this, a discursive analysis of 200 public and official documents was carried out, which gather contest bases for the awarding of scholarships, forms and instructions to distribute resources for research. The documents analyzed are understood as a representation of the public management of science, but above all as objects of order and performance in the scientific world. After a discursive analysis of the documents, we can show how the instruments trace the development of a "quantified academic self"; which requires that academics become actively involved, by registering their activities under the language of measurement and quantification. Without a doubt, the translation of scientific activity into this type of device plays a transcendental role, transforming scientific lives. The article shows how documents act on subjectivity and concludes by reiterating the need for a critical use and commitment to management technologies in public science policies.

Keywords: Management instruments; work subjectivity; capitalism academic

\section{RESUMO}

Este artigo reflete sobre a subjetividade dos acadêmicos a partir de uma análise empírica qualitativa dos instrumentos de ação pública para gerir a produção científica no Chile. Este estudo propõe que os dispositivos de gestão não só 
procuram otimizar a produção científica nacional, mas também têm efeitos importantes sobre aqueles que desenvolvem o conhecimento. Para investigar isso, foi realizada uma análise discursiva de 200 documentos públicos e oficiais, que reúnem bases de concursos para a concessão de bolsas, formulários e instruções para distribuição de recursos para pesquisa. Os documentos analisados são entendidos como uma representação da gestão pública da ciência, mas sobretudo como objetos de ordem e desempenho no mundo científico. Após uma análise discursiva dos documentos, podemos mostrar como os instrumentos traçam o desenvolvimento de um "eu acadêmico quantificado"; o que requer que os acadêmicos se envolvam ativamente, registrando suas atividades sob a linguagem de medição e quantificação. Sem dúvida, a tradução da atividade científica para este tipo de dispositivo desempenha um papel transcendental, transformando vidas científicas. O artigo mostra como os documentos atuam sobre a subjetividade e conclui reiterando a necessidade de um uso crítico e compromisso com as tecnologias de gestão nas políticas públicas de ciência.

Palavras-chave: Instrumentos de gestão; subjetividade laboral; academic capitalismo

\section{La subjetividad académica y la gestión basada en números}

$\mathrm{D}$ urante las últimas tres décadas, las Políticas Públicas (PP) para las ciencias nacionales han vivido profundas transformaciones, lo cual se enmarca en un giro mundial en las formas estatales de gestionar la producción de conocimiento (Slaughter \& Leslie, 1997). Dichas PP en ciencias se orientan por el llamado New Public Managment (NPM). EI NPM o Nueva Gestión Pública ha buscado gestionar de manera eficiente y con probidad los recursos del Estado, impactado en la mayoría de los servicios públicos, tales como educación, políticas sociales y salud entre otros. Particularmente en el área de la ciencia este aire de eficiencia se ha concretado en acciones tales como la descentralización y diversificación de las fuentes de financiamiento del trabajo científico, incorporación de la competencia como el principal mecanismo regulador, instalación de una cultura del accountability y desarrollo de sofisticados instrumentos de acreditación y medición de los procesos (Slaughter y Leslie, 1997). 
Bien es sabido que esta nueva forma de gestionar la ciencia no puede avanzar sin la voluntad de las comunidades académicas (Salmi, 2009). Por esta razón, la vida académica de científicos y científicas, la reorganización de su actividad, y como es que la ética y la subjetividad son una condición para el desarrollo exitoso de las nuevas regulaciones de la ciencia en Chile están en el centro del debate. En el panorama nacional descrito, instituciones gubernamentales, universidades y centros de investigación han innovado en dispositivos de gestión sofisticados para conducir, registrar y monitorear la fuerza laboral académica y la producción científica de los cuerpos científicos. De esta manera se han masificado con sorprendente naturalidad instrumentos como rankings de productividad investigativa, indicadores de citación, bonos por publicación y fondos de investigación concursables, entre otros. Sin duda la incorporación de estos instrumentos, se apareja al acelerado y creciente uso de tecnologías de la información.

En efecto las tecnologías de gestión, objeto de este estudio, son justamente los dispositivos de registro, medición y cálculo de aspectos generales y específicos del trabajo académico y universitario (Lim, 2019; Moore \& Robinson, 2016). Estos instrumentos son centrales para el funcionamiento de la ciencia neoliberal, ya que permiten la instalación de una cultura basada en el telecontrol, la auditoria, y a la vez que legitiman un modelo de gobernanza meritocrático de aparente probidad. En definitiva los instrumentos parecen ofrecer una lectura imparcial del mundo del trabajo, lo cual permitiría gestionar los recursos institucionales, asegurar la calidad de sus servicios y asignar presupuestos con eficiencia, basada en evidencias numéricas. La Nueva Gestión Pública se engarza cómodamente con las nuevas tecnologías y de esta manera los dispositivos de cuantificación se han instalado rápidamente en diversas entidades tales como gobiernos, instituciones universitarias y consultoras. De acuerdo con Miller \& O’leary (2007) es importante poner atención a estos mecanismos de gestión, en tanto se trata de núcleos densos de información donde intereses 
políticos, subjetivos y económicos se encuentran y coordinan. Afortunadamente la masificación del uso de los instrumentos de cuantificación se ha acompañado de un creciente interés por investigar sobre las consecuencias del uso de Number based gobernance (Williamson, 2016). Este estudio se pregunta por la subjetividad laboral y la relación del trabajador consigo mismo, promovida en estos cuadros de gestión y sostenidas por las tecnologías basadas en números y mediciones. Moore y Robinson (2016) hablan de un "quantified self" en el mundo del trabajo. ¿Podremos reconocer algo así en el trabajo científico? ¿De qué se trata este yo cuantificado? ¿Cómo funciona? El presente trabajo se suma a los esfuerzos por comprender el funcionamiento e impacto de la gestión basada en números y busca avanzar en una reflexión en torno a las consecuencias subjetivas en los trabajadores de la ciencia y las subjetividades laborales en general.

Este escrito muestra cómo la ciencia en Chile es un caso ejemplar en torno a la gobernanza basada en números en América Latina. Revisamos a partir de un Análisis del Discurso Pragmático el despliegue de estos instrumentos en el marco de la Nueva Gestión Pública. A continuación exponemos los resultados obtenidos del análisis pragmático de discurso a una muestra de 200 documentos oficiales relativos a las políticas públicas que organizan la ciencia en Chile (Prior, 2008; Potter \& Wetherell, 1987). Los resultados los hemos organizado en 3 categorías que describen el impacto de estos instrumentos sobre la subjetividad académica: a) Representación numérica del trabajador, b) Registro, monitoreo y autocontrol del trabajador y c) Parámetros y estándares como ideales para el trabajador. Las 3 categorías en su conjunto permiten caracterizar el funcionamiento de los instrumentos de cuantificación, a la vez que ofrecen claves para una lectura crítica acerca de la gestión de la ciencia y nuestra posición como científicos.

\section{La academia Chilena como caso ejemplar de la Nueva Gestión Pública}


Desde fines de la década de los 70 , las políticas públicas en Chile experimentan importantes transformaciones acordes a un contexto nacional de progresiva neoliberalización. En general una expresión de esto son las reformas de financiamiento de los servicios del Estado (Sisto, 2017). Un ejemplo particular es el caso de la educación superior, donde se disminuye el subsidio estatal y en paralelo se crean universidades privadas. De acuerdo con Bernasconi (2015) se impuso un sistema de competencia entre las instituciones públicas y privadas, como estrategia de sobrevivencia. Buena parte del gasto público en educación superior comienza a distribuirse mediante concursos y de esta manera diferentes instituciones, programas y académicos deben disputar los menguados recursos fiscales. En los años 90, con el advenimiento de la democracia este sistema no sólo es sostenido, sino perfeccionado: la competencia entre instituciones será explícitamente declarada como un mecanismo promotor de la eficiencia y los instrumentos de gestión se vuelven sofisticados a la hora de medir y controlar la justa competencia. Aparejado a una nueva estructura de financiamiento, la academia debe asumir una nueva lógica para organizarse y dar respuesta a esta inédita relación con el Estado. Esta reorganización de la Universidad confía en que más 'management' es indispensable para el éxito de las organizaciones públicas, incluyendo a las universidades (Fardella, Sisto, \& Jiménez, 2015). De esta manera el paradigma gerencial se asienta en las instituciones universitarias.

Es posible señalar que las transformaciones vividas por la universidad chilena encuentran su fundamento en dos lógicas propias de los estados neoliberales: La nueva gestión pública (NPM) y la Política Basada en Evidencias (EBP). La primera refiere a una forma de administración estatal, inspirada en la empresa privada, cuya implementación se ampara en el argumento de enmendar la densa burocracia, mediante tecnologías eficientes y competitivas (propias de la organización privada). Una expresión de estas ideas son descentralización y diversificación de las fuentes de financiamiento para la universidad (y otros servicios en general); el mercado como el 
principal mecanismo regulador a la vez que aseguramiento mínimo de la calidad de los servicios mediante instrumentos de acreditación. A su vez, estos mecanismos de acreditación de calidad van favoreciendo la expectativa de una cultura de accountability y eficacia para los procesos de trabajo (Fardella et al., 2015). Esto se traduce en la comprensión de las instituciones universitarias como organizaciones prestadoras de servicios de formación (profesional y avanzada), de investigación y asistencia técnica. Las cuales, para aumentar su productividad y calidad de servicio deben ser puestas a competir entre ellas, lo que estimularía a las instituciones a conectarse con las diversas demandas del sector empresarial, de potenciales estudiantes, del gobierno y otros posibles clientes.

La segunda (EBP), se instala bajo la promesa de mejorar el desempeño de la administración estatal mediante el uso de información de alta calidad. La alta cualificación de la información estaría dada por fuentes tales como conocimiento especializado, investigación nacional e internacional, datos estadísticos, stakeholders y think tanks (Head, 2010). En coherencia con esto, para fundamentar normativas y regulaciones debe contarse con la mejor evidencia disponible (Castillo-Sepúlveda, 2019; Head, 2010), relevando la información de índole científica, el registro sistemático de acciones y el cálculo de prácticas y eventos a través de diversos manuales e índices. Siguiendo a Castillo-Sepúlveda (2019), ello no solamente comporta la formulación de una retórica que recurre a fuentes de información "confiable" para la promulgación de políticas, sino también incide en la construcción de instrumentos que permitan la producción de los datos para tomar decisiones basadas en ello (CastilloSepúlveda, 2019; Head, 2010).

Las políticas públicas científicas, en consonancia con estas nuevas lógicas se orientan a perfeccionar un sistema de indicadores que deben organizar y visibilizar la acción de la academia (OCDE, 2009; Salmi, 2009). Esto empujará la proliferación de un conjunto de indicadores en la ciencia que permiten una gestión de procesos complejos, basada 
en evidencia: número de proyectos adjudicados en concursos de Comisión Nacional de Investigación Científica y Tecnológica (principal organismo chileno de financiamiento de investigación científico), montos adjudicados en proyectos concursables; número de publicaciones en revistas indexadas en las bases Web of Science-ISI, Scopus y SciELO, patentes solicitadas; proyectos de emprendimiento con financiamiento, etc.

Como han planteado Slaughter y Leslie (1997), estos indicadores, lentamente se constituyen a nivel local en los objetivos en torno a los cuales la academia se organiza (por sobre lo que las comunidades académicas pudiesen decidir colegiadamente), en tanto la adscripción y organización de la vida universitaria a estos indicadores es condición de financiamiento. Así, bajo la lógica del desempeño y los resultados, se han impuesto normas económicas y políticas vinculadas a la efectividad institucional que privilegian resultados por sobre cómo estos han sido producidos (Deem, Hillyard, \& Reed, 2007).

Frente al panorama descrito, diversos autores han descrito una transformación del ethos académico reorientando su acción formadora y de producción de conocimientos a aquello que pueda ser vendido a estudiantes y empresas o que se vuelva visible para rankings e indicadores de diversas mediciones. Tanto el NPM como la EBP presentan una gramática compleja que tensiona el espíritu tradicional de la academia y sin duda demanda nuevas subjetividades donde cimentar los cambios (Deem et al., 2007; Fardella et al., 2015).

\section{Instrumentos de cuantificación, managment y subjetivación}

Actualmente podremos notar un relativo consenso en el vínculo entre managment y procesos de subjetivación. La definición de esta relación puede ser fácilmente aceptada al aseverar que las formas de gestión conforman un cuerpo de saberes e instrumentos por medio de las cuales se busca organizar la fuerza laboral. De esta 
manera, la gestión puede entenderse como un conjunto de prescripciones y demandas para los individuos en sus trabajos o espacios productivos (Sisto, 2017; Soto, Fardella, Valenzuela, \& Carvajal, 2017).

Evidentemente aceptar esta relación comporta una noción particular de subjetividad, donde ésta debe ser comprendida como una conformación sociohistórica y materialmente anclada. En este sentido, la dimensión subjetiva demarca una interfaz entre el gobierno de los sujetos y los sujetos, corporeizando la acción managerial. Tal como señalan Potter y Wetherell (1987) las personas y las culturas se construyen mediante los mismos materiales, por tanto analizar una cultura y analizar la subjetividad de una persona son actividades similares entre sí. Al mismo tiempo esta conceptualización supone que ciertos aspectos del individuo y del proceso de individuación son potencialmente registrables bajo las coordenadas económicasproductivas actuales.

Un tercer elemento que acompaña la relación subjetivación y managment son los instrumentos de gestión que participan sosteniendo vínculo. Es cierto que la consideración de los instrumentos y tecnologías como agentes en la organización del trabajo no es nueva. Tal cual fue estudiado en los estudios de Tavistock y los "sistemas socio-técnicos". Sin embargo lo "socio" y lo "técnico" han sido estudiados con relativa independencia, aun cuando se reconoce su mutua influencia (Nicolini, 2012; Latour, 2011). Si bien en las últimas décadas hay un giro hacia la comprensión más amplia de los artefactos de gestión, estos han tenido un interés más bien pragmático y de optimización. Puntualmente, Orlikowski y Scott en el 2008 analizaron todos los artículos publicados en las principales revistas de gestión en la década anterior y encontraron que en su mayoría (el 95\%) no consideran el papel y la influencia de la tecnología en la vida organizacional. De acuerdo a esto parece ser que la reflexión en torno a la interacción entre lo tecnológico y lo subjetivo ha ido quedando descubierta e inexplorada. 
En este sentido Latour (2011) y otros autores han recuperado la atención en lo material y en los artefactos tecnológicos, invitando a tomar una nueva perspectiva analítica sobre los fenómenos laborales. Este impulso ha conformado un tipo de estudios organizacionales con enfoque performativo (Gherardi, 2006; Nicolini, 2012), que han enfatizado en la importancia de considerar la subjetividad, la tecnología, los cuerpos, la estética como elementos de igual importancia que los procesos organizacionales. En este sentido, la gestión y los procesos subjetivos se tejen y articulan con artefactos y materiales que van configurando prácticas y experiencias organizacionales socio-tecnológicas. Esto permite un avance epistémico notable respecto de los primeros enfoques que intentaron incorporar lo material y tecnológico al estudio del trabajo y la gestión. Entonces se trata de una renovada sensibilidad hacia las tecnologías de la gestión, situándolas en una relación de simetría con lo subjetivo y simbólico. En definitiva, es una perspectiva performativa de las nuevas tecnologías de gestión, donde se las piensa como objeto y materialidades, que no son instrumentos, sino actores de los procesos de trabajo. En pocas palabras las tecnologías sociomateriales importan, porque generan consecuencias más allá de las que han sido pensadas en su diseño, por tanto, se vuelve necesaria una dimensión de análisis ético, que permita pensar y evaluar su actuar. Este enfoque provee entonces un pool de herramientas e ideas para comprender e imaginar las posibilidades de actuar y efectos de las actuales tecnologías en los espacios laborales. Al mismo tiempo que resulta particularmente útil para mirar las transformaciones del trabajo académico y las nuevas tecnologías implementadas para organizarlo y gestionarlo.

En coherencia con estos estudios se ha desarrollado una conceptualización especifica en torno a las tecnologías de cuantificación, lo cual amplia de manera creativa los estudios en torno a la gestión (Lim, 2019). Se engloba bajo el genérico de dispositivos de cuantificación un conjunto de tecnologías que tienen por función medir, bajo una premisa no explicita de que la medición sistemática permite una valoración diáfana del 
mundo y sus personas. De esta forma, los dispositivos de cuantificación podríamos definirlos como tecnologías para el registro y cálculo sistemático de cualquier experiencia/fenómeno humano o también no humano (Lupton, 2016). La aparición de estos instrumentos en el mundo del trabajo ha sido explosiva y esto pareciera estar facilitado por las características actuales del trabajo, como la inapelable incorporación de dispositivos computacionales, condiciones flexibles de trabajo, telepresencia, justin-time y outsourcing (Moore \& Robinson; 2016). Asociado a este contexto y a la alta complejidad de gestionar tales procesos productivos, los instrumentos de cuantificación realizarían un aporte importante en la gestión pública (Sisto, 2017). Justamente la capacidad de estos instrumentos para cuantificar y medir es lo que les permitiría transformar procesos complejos y heterogéneos, en indicadores simples de carácter numérico (Miller y O'Leary, 2007). Se trata de dispositivos que permiten que procesos multidimensionales de compleja gestión, en particular del área productiva y laboral, puedan ser utilizados mediante representaciones simples y manejables. En efecto, las tablas y los indicadores condensan información acerca de elementos y vínculos heterogéneos y dispares, que son representados con una aparente homogeneidad en tablas de almacenaje de información y fácil interpretación para quienes deben tomar decisiones. De esta manera, grandes volúmenes de información derivados de la gestión pública, se convierten en unidades controlables que permitirían la comparación y seguimiento de diferentes unidades productivas (como la fuerza laboral).

Ligada a la capacidad de representación de los instrumentos de cuantificación encontramos la función performativa. Acorde a Callon (1998) los podemos describir como objetos con agencia en tanto la función de representación, se acompaña de la distinción y cualificación de determinados aspectos del trabajo y del trabajador. En concreto los indicadores, las tablas, los rankings, no solo representan en términos 
simples el estado de una cuestión, sino que además portarán demandas a la acción, referencias e ideales para quienes trabaja, performando la escena laboral cotidiana. Como hemos señalado previamente, los instrumentos de cuantificación en el ámbito de la gestión no son sólo sistemas de representación, sino que hablamos de instrumentos de mediación puesto que enlaza la acción entre subjetividad y el proceso productivo. Esta coordinación permanente, se produce toda vez que el trabajo de individuos debe moldearse y ser traducible para calzar en el lenguaje calculativo de estos instrumentos. Estos instrumentos constituyen así una gramática en la cual deben inscribirse las diferentes actividades de los académicos. En efecto, la gestión por incentivos al desempeño y las métricas y tecnologías numéricas son un ensamble funcional, donde estos dispositivos (entre otros asuntos), facultan escenarios laborales de constante vigilancia y de intensa competencia entre los trabajadores (Soto et al., 2017).

En el escenario académico-científico, la participación de las tecnologías de cuantificación y medición, así como las interpelaciones a los investigadores para medir y gestionar su propia productividad son evidentes. Rankings, indicadores de citación, índices de internacionalización no solo dibujan el paisaje científico sino que sostienen la sofisticada tarea de engarzar la vida cotidiana y los individuos al tele gobierno. Este escenario faculta la pregunta que orienta este trabajo y justifica nuestros esfuerzos por comprender el funcionamiento e impacto de la Gestión Basada en Números y busca avanzar en una reflexión en torno a las consecuencias subjetivas en los trabajadores de la ciencia y las subjetividades laborales en general.

\section{Método}

El material que compone nuestro corpus consta de 200 documentos oficiales provenientes de diferentes unidades gubernamentales implicadas en la gestión y financiamiento de la ciencia. Este material incorpora llamados a concursos a diversos 
fondos de investigación, bases de postulación a becas y presentaciones oficiales de programas gubernamentales. De acuerdo con Íñiguez y Antaki (1994), entendemos la representatividad no desde la perspectiva numérica sino en su potencialidad para mostrar heterogéneas versiones de nuestro objeto de estudio.

Este análisis opera bajo el supuesto de que los documentos analizados constituyen discursos semióticos materiales que portan fórmulas para leer e interpretar la actividad científica y académica. Es decir, los textos analizados a la vez que regulan el trabajo científico, definen y valoran formas de ser y hacer ciencia.

Para abordar el objetivo planteado hemos utilizado un Análisis del Discurso pragmático (Potter y Wetherell en 1987). Bajo este marco nuestro análisis consta en identificar repertorios interpretativos, entendidos como formas discursivas recurrentes para construir realidad. Para nuestra investigación buscaremos formas en que los documentos de gestión de la ciencia construyen y empujan determinadas formas de ser sujeto científico. Es decir ¿qué subjetividad y relación consigo mismo promueven y sostienen los instrumentos de cuantificación propio de los actuales sistemas de gestión?.

Bajo esta pregunta hemos fragmentado los textos buscando unidades de significado que abordaran implícita o explícitamente al académico y su quehacer, y tuvieran efectos discursivos en la constitución de este. La identificación de fragmentos y codificación de estos mismos se realizó con el apoyo del software de análisis de datos cualitativos ATLAS.TI, a modo de facilitar la gestión de los datos.

\section{Resultados}

Una vez organizado el material agrupamos los códigos en 3 categorías, comprendidas como 3 diferentes consecuencias que tienen los instrumentos de cuantificación en el proceso de subjetivación. A saber: a) Representación numérica del trabajador, b) 
Registro, monitoreo y autocontrol del trabajador y c) Parámetros y estándares como ideales para el trabajador.

\section{La representación numérica del trabajador}

El número, la cantidad y el procedimiento algorítmico, se presenta y ocupa un lugar central en los documentos revisados. El número, arrojado por registros y mediciones particulares son presentadas en los textos analizados como unidades de información que permitirían plasmar la actividad del científico. Los documentos analizados, en su conjunto funcionan como piezas que componen un mismo lenguaje; constituyéndose como elementos lingüísticos que colaboran entre sí para conformar representaciones numéricas complejas y dinámicas del trabajador.

A continuación, se ilustra esto con un fragmento que corresponde a un segmento de las bases del principal concurso nacional de financiamiento a la investigación. El texto introduce los criterios de elegibilidad de los concursantes, entre ellos la productividad de investigador y señala:

Se evaluará la productividad científica del (de la) Investigador(a) Responsable del proyecto, no así de los (las) Co-investigadores(as). Los antecedentes serán evaluados por el Grupo de Estudio en el cual quede adscrito el proyecto y considerará un máximo de 10 publicaciones informadas en la postulación (artículos en revistas científicas, libros y/o capítulos de libros) aceptados, en prensa o publicados a partir del año 2013 (Comisión Nacional Científica y Tecnológica, 2019a, p. 14)

El texto, mediante la indicación de los criterios de evaluación de la 'producción' del investigador ('artículos en revistas científicas, libros y/o capítulos de libro') restringe el espectro de outputs científicos posibles y actividades tales como conversatorios, peer review, formación de investigadores o divulgación científica son desestimadas en el registro y conteo de la producción. Adicionalmente, se establece un rango de tiempo 
para el registro de la productividad (5 años). Es decir, una actividad científica se volverá admisible para su registro y cálculo sólo si ha sido desarrollada en los últimos 5 años, instalando un ritmo y la necesidad de mantenerlo para continuar vigente en la competencia.

Junto a la jerarquía de actividades, definición de ritmos de trabajo, el fragmento ilustra un asunto no menos importante: la concepción de autoría. De acuerdo a la asignación de puntaje en la tabla, el primer autor es el fundamental y se asume que quién va en segundo lugar ha realizado una contribución menor, así mismo es interesante destacar que el primer autor obtiene el mismo valor que ser autor único. De esta manera el valor de cada contribución está determinado por regulaciones externas al equipo de autores. Lo que se pone en cuestión acá no es el orden de autor, sino la prescripción de un tipo de subjetividad que asume individualmente el trabajo científico. Ratificando una premisa fundamental del managment contemporáneo: el trabajo es una actividad individual, y debe ser medido e incentivado como tal para favorecer la competencia. Con ello se asume la irrelevancia de otras subjetividades laborales, que proceden de manera colectiva o que se sostienen con ideales contrapuestos a la competencia, como la colaboración, la sororidad o el cuidado de otros. Al mismo tiempo a esta subjetividad individualizada la acompaña un entorno borroso, es decir la omisión de contexto o condiciones de la producción académica (condiciones laborales, micro políticas institucionales, dimensiones de género, equipos de trabajo, etcétera). Tal como lo ilustra el fragmento de Principales indicadores Cienciométricos indicados por el Observatorio de Ciencia, Tecnología, Innovación y Emprendimiento (2014) cuando define la innovación como conocimiento innovador y este es traducido como "Número de trabajos publicados por un dominio dado y citada en las patentes" (p. 278). Sabemos que la innovación es un proceso complejo, que tiene que ver con el capital social que permiten y reconocen procesos creativos, con sistemas históricos, sociopolíticos y económicos y no con una habilidad personal que actúa sobre un 
dominio específico. Según Kapur (2007), el régimen neoliberal idealiza a los trabajadores creativos, explotando sus cualidades, en tanto atributos individuales, desconociendo las dimensiones sociales y contextuales del trabajo. Salmi (2009) ha nominado este fenómeno 'voluntarismo mágico', respondiente a una ideología que elude una realidad de exclusiones, competencias, precariedad de las condiciones laborales al insistir en una capacidad milagrosa de cada individuo. Y la creencia ciega en que los logros y desempeños de los trabajadores se corresponde a una capacidad del sujeto y no de la organización (Moore, \& Robinson, 2016).

El fragmento ilustra cómo los documentos, no solo restringen el abanico de actividades de un académico y marcan los ritmos de producción, sino y sobre todo lo que observamos son tecnologías de cuantificación que demarcan al investigador como sujeto individualizado y por tanto hiper responsabilizado de su producción científica y su devenir como científico (Lim, 2019). De esta manera los fenómenos propios de la producción científica son descritos como acciones que se sitúan en el ámbito de la agencia, el interés y el mérito humano.

Por tanto las mediciones a la vez que informan sobre el estado de alguna cosa, también demarcan lo medible, de esta manera los cálculos acerca de nuestro trabajo diferencian las tareas productivas de las 'no productivas', a la vez que establecen una jerarquía entre ellas. Con ello lentamente las actividades 'no productiva' se van extinguiendo, los procesos que toman más tiempo son evadidos. $Y$ en su reemplazo protagonizan la jornada laboral, aquellas actividades que maximizan un índice que muestre un yo productivo. En paralelo, el trabajo individual desplaza el trabajo colectivo, en tanto la consolidación de un perfil competitivo tendrá mayores beneficios en la carrera científica que actividades que favorezcan la comunidad. La fragmentación de fenómenos complejos en diferentes artificios medibles es una de las operaciones más comunes de estas tecnologías de la cuantificación, tal como se observó en los documentos analizados. Sin embargo, cabe señalar que este 
procedimiento no se trata de un cálculo que dé cuenta auténtica e íntegramente, de la cualidades de los fenómenos (si se pudiese tal cosa), más bien se trata de un proceso arbitrario de relevar ciertos aspectos de la producción académica por sobre otros. Tal como se puede apreciar en los fragmentos citados.

Cuando los sistemas de accountability ofrecen lockers para registrar el trabajo, lo restringen al suponer un número limitado de tareas para ser notificadas. Este procedimiento presume consensos al tomar opciones discrecionales, sin embargo invisibiliza los criterios con los cuales opera. Sumado a esto, el carácter oficial de los documentos -en tanto bases para un concurso que condiciona financiamiento para investigar- favorece su carácter prescriptivo y orientador de las acciones de los trabajadores. De esta manera la representación sostenida y vehiculada por los indicadores, termina por volverse la única representación válida del científico. De aquí que el nuevo sistema de gestión solo visibilice una forma subjetiva. Lamentablemente la distancia entre legible y legitimo es mínima. Y por este motivo la manera legitima de obtener financiamiento empuja a los científicos a adscribirse e inscribirse a sí mismo en este nuevo lenguaje. De este modo los documentos producidos por las nuevas regulaciones, constituyen un código imperativo de inteligibilidad que ordena, organiza y produce determinadas subjetividades laborales.

\section{Registro, monitoreo y autocontrol del trabajador}

Como ilustramos en el apartado anterior los dígitos, cantidades, cálculos e indicadores conforman una representación particular del yo académico, provisionada de contenidos arbitrarios con efectos para la subjetividad laboral de los académicos. En consecuencia, si un investigador quiere mostrarse productivo y competir por fondos para llevar a cabo tareas propias de su rol (investigar), debe catalogar e inscribir su trabajo en las categorías que las nuevas regulaciones han dispuesto para ello. De esta forma, su trabajo será leído como productivo. 
Los documentos analizados van estableciendo categorías y métricas que definen y ordenan diferentes aspectos del trabajador académico. Como revisamos en el apartado anterior los instrumentos de cuantificación no solo registran información en forma de número, sino que la representación viene cargada con un peso específico, que le dará valor en comparación a un conjunto de otros elementos. Esto se ilustra con claridad cuando las bases oficiales exponen los criterios de evaluación curricular Fondecyt 2020, señalando por ejemplo las equivalencias de puntaje entre cada producto "Libro monográfico publicado por editorial con referato externo: 1.00; Artículo en revista indizada en WOS, Scopus o ERIH: 0.75 (...) Artículo en revista con otra indización y con revisión de pares: 0.20" (Comisión Nacional Científica y Tecnológica, 2019c, p. 26). Sucede que de esta misma manera se le asigna valía a diferentes dimensiones del sujeto, estableciendo jerarquías entre cualidades y finalmente construyendo un ideal que funciona como referencia para el trabajador.

Así mismo el ideal funciona como un parámetro organizador de los trabajadores. Como ha determinado Coin (2019), se trata de una economía reputacional, donde la motivación de cada trabajador o unidad productiva debiera compararse con otras subjetividades cuantificadas y alcanzar las primeras posiciones del ranking. Un académico que quiera conseguir fondos para realizar su trabajo, debe necesariamente adecuarse y traducirse así mismo a este lenguaje, convertirse en el modelo y ocupar las posiciones superiores del ranking.

La posición que ocupa un determinado "yo cuantificado" se interpreta como una representación justa del mérito y la competencia, simulando proporcionar información neutra y objetiva sobre las capacidades humanas desplegadas en este trabajo, aun cuando estos datos son una elección arbitraria acerca de cómo puede o deber ser un académico. Numerosos autores (Lupton, 2016; Moore y Robinson, 2016) han documentado que estos mecanismos de gestión fomentan una imagen cuantitativa del trabajador que no necesariamente calza con la autoimagen o posibilidad de 
rendimiento del trabajador. Este gap entre el ideal referencial y la imagen de sí que ofrecen los instrumentos de cuantificación, generarían una sensación de inadecuación permanente y de falta de cumplimiento, cofirmando a la vez la desvalorización de aquellas formas subjetivas subalternas de ser académico.

Coin (2019) describe la relación entre la experiencia de inadecuación y la lucha competitiva neoliberal, donde la universidad y el mundo productivo en general encarna gran parte de la responsabilidad de cernir a la población de trabajadores. Esto es evidente en uno de los fragmentos recogidos de la base de la iniciativa núcleo milenio 2017 donde se expone:

La escala de evaluación a utilizar por los evaluadores en todas las etapas del concurso será a siguiente:

Calificación entre 100 y 199, equivalente a sobresaliente (outstanding).

Calificación entre 200 y 299, equivalente a bueno (good)

Calificación entre 300 y 399, equivalente a razonable, pero requiere importantes mejoras (fair but requires important improvements).

Calificacion entre 400 y 500, equivalente a No competitivo (non competitive).

(Iniciativa Milenio, 2019, p. 28)

De esta manera, los instrumentos de cuantificación funcionan como un dispositivo de criba, es decir se trata de filtros de información que en ningún caso contribuyen a aumentar la calidad del conocimiento, como un dispositivo para la selección y ordenamiento de los individuos según sus capacidades útiles al sistema productivo económico.

Los instrumentos de cuantificación son claves en la gestión contemporánea para filtrar y ordenar a los trabajadores desde una actitud escéptica y aparentemente desideologizada. En tanto, la expulsión social queda inscrita como una incompetencia o insuficiencia. De cierta forma la excelencia o las mejores posiciones en el ranking, así como el fracaso o la expulsión del sistema por cumplir o no los estándares y 
parámetros son construidos como una consecuencia de la conducta y voluntad individual, aun cuando son resultado de una forma de gobierno propias de las actuales organizaciones del trabajo.

\section{Parámetros y estándares como ideales para el trabajador}

Un aspecto interesante en este análisis es mirar cómo los dispositivos de cuantificación son fundamentales para gobernar la relación que los sujetos sostienen consigo mismo o dicho de otro modo para hetero conducir el autocontrol. ¿Cómo funciona esto?

Lo primero que debemos apuntar es el ejercicio de registro que cruza transversalmente todos los documentos analizados. Con esto queremos decir que todos los dispositivos de cuantificación requieren indefectiblemente ser provisionados de datos para proceder con sus algoritmos y esta tarea ha sido naturalmente asignada al académico. En el caso de los dispositivos de cuantificación el registro constituye un ejercicio permanente de volcamiento de información acerca de sí en los lockers que los formularios o software ofrecen para ello. Así se ilustra en el fragmento:

Para la presente postulación deberá completar este formulario y cargarlo en el sistema de postulación en línea. (...) A continuación deberá completar toda la información que se solicita. Todos los campos con * son obligatorios. En caso de faltar cualquier dato obligatorio, la presente postulación será declarada inadmisible (Comisión Nacional Científica y Tecnológica, 2019b, p. 2).

Como es suponible, cada investigador no puede informar voluntariamente lo que quiera de sí, limitando la información a aquello por lo cual es interrogado. Con esto los instrumentos de cuantificación aseguran que la información que cada sujeto ha entregado se vuelva estandarizable, comparable y por tanto rankeable. Una vez ingresados los datos a la caja negra de los algoritmos producirán un indicador, una cifra que indica algo sobre quien cuidadosamente ha ingresado sus datos. Se trata de 
una nueva representación de sí validada por el sistema. Esta representación del trabajador vuelve a él como una autoimagen, que simula autenticidad aun cuando ha sido moldeada por cálculos y procedimientos numéricos. De acuerdo a los textos revisados, se trata de instrumentos de cálculo que poseen la particularidad de mediar objetivamente entre el sujeto y la propia percepción de sí. Por mediar comprenderemos la maniobra de demarcar los heterogéneos aspectos que componen la realidad de la subjetividad laboral, a la vez que diseñar la relación entre ellos. Por ejemplo, media la relación que debe establecer el científico con el tiempo (Comisión Nacional Científica y Tecnológica, 2019b), o como interviene la relación entre el autor y para quien este desee escribir, al otorgarle mayor valor en revistas indexadas de bases de datos privadas.

Si el académico quiere dejar una huella de sí competitiva, a la vez que conseguir una representación de sí positiva deberá inscribir la mejor versión de sí en el sistema. La presentación de sí que debe ser construida por los usuarios de los sistemas de cuantificación debe ser una representación numérica que logre entrar en competencia con representaciones de otras subjetividades que también se han adscrito y traducido al mismo lenguaje numérico estandarizado. De esta manera las tablas, los indicadores y los sistemas de conversión estimulan a que los académicos trabajen por la versión más productiva de sí mismos, a la vez que sostengan en el tiempo esta imagen mediante la permanente producción de diferentes outputs validados. Esto implica al menos dos cosas: llevar a cabo acciones que le permitan esto (publicar en nuestro caso) y segundo establecer una relación de exigencia, esfuerzo, monitorización y autopromoción de sí mismo.

De esta manera los instrumentos revisados fomentan la relación de autogestión o auto explotación. Los dispositivos acá mencionados facilitan que los académicos se administren a sí mismos como una fuerza laboral. Mediante las representaciones que los cálculos construyen acerca del trabajador/trabajo los mismo individuos pueden 
continuar registrando su actividad, monitorearlas e introducir los cambios correspondientes. Es interesante mostrar como estos artefactos numéricos proceden, colándose en el alma y modificando a su vez, la relación de los académicos consigo mismo, situando al vigilante y trabajador dentro del mismo cuerpo. Se trata entonces de dispositivos de cuantificación que ocupan un lugar clave para gobernar sigilosamente la fuerza laboral académica, en tanto al prescribir la relación que el sujeto establece consigo mismo busca organizar heteronormativamente su subjetividad. Tal como señala Moore y Robinson (2016), se trataría de un autocontrol heterocontrolado y en este procedimiento, los instrumentos de cuantificación resultan un engranaje fundamental, pues delegará en el académico el registro, monitoreo y control sobre su propia fuerza de trabajo. Así el cálculo funciona como una gramática que ordena y regula la manera en que el sistema se colará en el sí mismo.

\section{Consideraciones Finales}

Mediante un análisis pragmático de discurso a documentos oficiales tanto de nivel estatal, Consejo Nacional para el Desarrollo de la Ciencia y la Tecnología (Conicyt) e institucional (de las propias Universidades), este artículo muestra cómo los instrumentos de cuantificación propios de la actual forma de gestionar la vida académica portan mandatos que modelan al sujeto académico. El yo cuantificado se enlaza con un funcionamiento general acerca de cómo funciona la producción neoliberal y sus sujetos (Coin, 2019). No es posible omitir el contexto sociohistórico de competencia como mecanismo de cohesión social, donde la lógica acumulativa del capitalismo académico y la transformación del ego y el prestigio en formas de capital nutren la escritura frenética de artículos para la industria académica.

Justamente son los instrumentos de cuantificación y el cálculo de nuestra actividad y de aspectos de nuestro yo laboral lo que habilita este sistema de producción, 
conduciendo hacia una creciente legitimación de los sistemas de cuantificación en la gestión del trabajo científico.

El trabajo presentado son los resultados iniciales de un trabajo en progreso, no obstante, se constituyen en un fuerte incentivo para reflexionar sobre el creciente y generalizado uso de la tecnología digital para gestionar la producción científica a nivel nacional. Urge realizar un mapeo algo más denso y sensible a las diferencias en torno a cómo operan las tecnologías involucradas en la captura y el registro de la actividad científica. Así mismo e igualmente importante es continuar reflexionando acerca de cómo operan estas tecnologías, pero sobre todo, como ha sido la respuesta de los académicos y su dócil engarce con las nuevas tecnologías.

Evidentemente es necesario analizar la consistencia de los cálculos en relación a aquello que suponen medir, pero sobre todo debemos pensar en los efectos subjetivos, intersubjetivos e institucionales que estas formas de gestión catalizan en las comunidades disciplinares y científicas. Si la ciencia, el conocimiento y los científicos han tejido un compromiso a lo largo de la historia con las sociedades contemporáneas para promover el progreso mediante el razonamiento y la lectura crítica; las actuales formas de conducir la producción científica y sus trabajadores deberán volver a mirar su capacidad para cumplir con este compromiso. Por ello parece de crucial importancia indagar en nuestras prácticas científicas y formas de interactuar con los dispositivos presentados.

\section{Agradecimientos}

Este trabajo se benefició del apoyo financiero del Proyecto Fondecyt Regular 1180129 "El trabajo científico en Chile" y de la Iniciativa Científica Milenio del Ministerio de Economía, Fomento y Turismo de Chile adjudicado al Centro Núcleo Milenio Autoridad y Asimetrías de Poder. 


\section{Referencias}

Bernasconi, A. (2015). La educación superior de Chile: Transformación, desarrollo y crisis. Santiago. Ediciones UC.

Callon, M. (1998). El proceso de construcción de la sociedad. El estudio de la tecnología como herramienta para el análisis sociológico. A Domènech, M. and Tirado. FJ Sociología simétrica. Ensayos sobre ciencia tecnología y sociedad. Barcelona: Gedisa.

Castillo-Sepúlveda, J. (2019). Gubernamentalidad y somatocracia en el Régimen de Garantías Explícitas en Salud en Chile. Estudios atacameños, (ahead), 0-0.

Coin, F. (2019). La ineptitud del digital academic: precariedad y salud en el mundo universitario. RECERCA. Revista de Pensament y Anàlisi, (24), 114-133.

Comisión Nacional Científica y Tecnológica, (2019a). Bases Concurso Nacional de Proyectos FONDECYT Regular 2019. Recuperado de https://www.conicyt.cl/fondecyt/files/2018/07/BASES-CONCURSO-REGULAR2019.pdf

Comisión Nacional Científica y Tecnológica, (2019b). Convocatoria Nacional Subvención a la Instalación en la Academia 2019. Recuperado de https://www.conicyt.cl/pai/files/2019/03/Formulario-Postulaci\%C3\%B3n-SIA2019.docx

Comisión Nacional Científica y Tecnológica. (2019c). Bases Concurso Nacional de Proyectos FONDECYT Regular 2020. Recuperado de https://www.conicyt.cl/fondecyt/files/2019/05/Bases-Concurso-FONDECYTRegular-2020.pdf

Deem, R., Hillyard, S., \& Reed, M. (2007). Knowledge, higher education and the new managerialism: The changing management of UK universities. Oxford: Oxford University Press. 
Fardella, C., Sisto, V., \& Jiménez, F. (2015). Nosotros los académicos. Narrativas identitarias y autodefinición en la universidad actual. Universitas Psychologica, 14(SPE5), 1625-1636.

Gherardi, S. (2006). Organizational knowledge: The texture of workplace learning. Oxford, UK: Blackwell Publishing.

Head, B. (2010). Reconsidering evidence-based policy: Key issues and challenges. Policy and Society, 29(2), 77-94.

Iniciativa Milenio. (2019). Base Tipo "Concurso de Núcleos Nuevos y Núcleos de Renovación del Programa Iniciativa Científica Milenio". Recuperado de http://www.iniciativamilenio.cl/wp-content/uploads/2017/11/Bases-nucleos-2017-12.pdf

Íñiguez, L., \& Antaki, C. (1994). El análisis del discurso en Psicología. Boletín de Psicología, 44, 55-75.

Kapur, J. (2007). "New" economy/old labour: Creativity, flatness, and other neoliberal myths'. In C. McKercher \& V. Mosco (Eds.), Knowledge workers in the information society. Lanham: Lexington Books.

Latour, B. (2011). "What's the story?" Organizing as a mode of existence. Recuperado de http://bruno-latour.fr/sites/default/files/110-ORGANIZATION-PASSOTH.pdf.

Lim, M. A. (2019). Governing Higher Education: The PURE Data System and the Management of the Bibliometric Self. Higher Education Policy, 1-16. Recuperado de https://link.springer.com/article/10.1057/s41307-018-00130-0

Lupton, D. (2016). The quantified self: A sociology of self-tracking. Cambridge: Polity Press.

Miller, P. \& O'leary, T. (2007). Mediating instruments and making markets: Capital budgeting, science and the economy. Accounting, organizations and society, 32(7), 701-734.

Moore, P., \& Robinson, A. (2016). The quantified self: What counts in the neoliberal workplace. New Media \& Society, 18(11), 2774-2792. 
Nicolini, D. (2012). Practice theory, work, and organization: An introduction. Oxford: OUP.

Observatorio de Ciencia, Tecnología, Innovación y Emprendimiento. (2014). Principales indicadores cienciométricos de la actividad científica chilena 2012 Informe 2014: una mirada a 10 años. Recuperado de http://ctie.economia.cl/wpcontent/uploads/2018/03/Indicadores-Cienciom\%C3\%A9tricos-2014.pdf

OCDE. (2009). La educación superior en Chile: Revisión de políticas nacionales de educación. Santiago: Ministerio de Educación.

Orlikowski, W. \& Scott, S. (2008). Sociomateriality: Challenging the Separation of Technology, Work and Organization. In W. Pasmore, Designing Effective Organizations: The Sociotechnical Systems Perspective (pp. 433-474). New York: Academy of Management Annals.

Potter, J., \& Wetherell, M. (1987). Discourse and Social Psychology. London: Sage.

Prior, L. (2008). Repositioning documents in social research. Sociology, 42(5), 821-836.

Salmi, J. (2009). The challenge of establishing world-class universities. Washington, D.C.: World Bank Publications.

Sisto, V. (2017). Gobernados por números: El financiamiento como forma de gobierno de la universidad en Chile. Psicoperspectivas, 16(3), 64-75.

Slaughter, S., \& Leslie, L. (1997). Academic Capitalism. Politics, Policies and the Entrepreneurial University. Baltimore: Johns Hopkins University Press.

Soto, Á., Fardella, C., Valenzuela, A., \& Carvajal, F. (2017). Las funciones performativas de los instrumentos en las redes de acción pública. Psicoperspectivas, 16(3), 7686.

Williamson, B. (2016). Digital education governance: data visualization, predictive analytics, and 'real-time'policy instruments. Journal of Education Policy, 31(2), 123-141. 


\section{Declaración de contribución de autores}

CF responsable del diseño e implementación de la investigación y proceso análisis de datos; $\mathrm{RZ}$ responsable producción y organización de toma de datos; $\mathrm{AC}$ del análisis de datos y edición del manuscrito. Todos los autores discutieron los resultados y contribuyeron a la versión final.

\section{Formato de citación}

Fardella, C., Navia, A.P.C. y Zavala, R. (2019). El académico cuantificado: La gestión performativa a través de los instrumentos de medición en la ciencia. Psicología, Conocimiento y Sociedad, 9(2), 77-103. doi: http://dx.doi.org/10.26864/PCS.v9.n2.15 